\title{
Clinico-laboratory Profile and Therapeutic Outcome of Serologically Confirmed Scrub Typhus in Children in Tertiary Care Children's Hospital of Nepal
}

\author{
Ravindra Kumar Sah ${ }^{1 *}$, Ram Hari Chapagain ${ }^{2}$, Sushan Man Shrestha ${ }^{3}$ and Ganesh Kumar Rai ${ }^{2}$ \\ ${ }^{1}$ National Academy of Medical Sciences (NAMS), Kathmandu, Nepal \\ ${ }^{2}$ Department of Pediatrics, National Academy of Medical Sciences (NAMS) and, Kanti Children's Hospital, Nepal \\ ${ }^{3}$ Department of Community Medicine, Institute of Medicine, Tribhuvan University, Nepal
}

*Corresponding author: Ravindra Kumar Sah, MD Resident (Pediatrics) National Academy of Medical Sciences (NAMS), Kathmandu, Nepal, Tel: +9779841675699; E-mail: ravindra.sah1989@gmail.com

Received date: July 09, 2018; Accepted date: December 21, 2018; Published date: January 01, 2019

Citation: Sah RK, Chapagain RH, Shrestha SM, Rai GK (2019) Clinico-laboratory Profile and Therapeutic Outcome of Serologically Confirmed Scrub Typhus in Children in Tertiary Care Children's Hospital of Nepal. Pediatric Infect Dis Vol.4 No.1:1.

Copyright: (c) 2019 Sah RK, et al. This is an open-access article distributed under the terms of the Creative Commons Attribution License, which permits unrestricted use, distribution, and reproduction in any medium, provided the original author and source are credited.

\section{Abstract}

Introduction: Scrub typhus is an acute febrile illness endemic to tsutsugamushi triangle including Nepal. The WHO identifies scrub typhus as a re-emerging disease in South-East Asia. Scrub typhus can have varied manifestations in children. We aimed to study clinicolaboratory profile and therapeutic outcomes of serologically confirmed scrub typhus in children.

Methods: A retrospective study was conducted at Kanti Children's Hospital, Nepal after obtaining ethical clearance from Institutional Review Committee, and reviewing the medical records of serologically diagnosed scrub typhus admitted to the hospital between August 2016 and January 2018. Relevant data were entered in and analyzed using SPSS 20.0.

Results: Children from 9 districts of Nepal severely affected by the 2015 earthquake were admitted; mostly from Dhading (14.5\%) and Gorkha (9.7\%) districts. All 62 children included in the study had a fever; whereas pain abdomen, vomiting, cough, headache, and generalized body swelling were present in $25.8 \%, 21.0 \% 24.2 \%, 14.5 \%$, and $14.5 \%$ cases respectively. Hepatomegaly, splenomegaly, hepatosplenomegaly, lymphadenopathy, and rashes were found in $53.2 \%, 38.7 \%, 32.2 \%, 17.7 \%$ and $9.7 \%$ cases respectively. Thrombocytopenia and hyponatremia were seen in $72.6 \%$ and $28.6 \%$ cases respectively. The combination of hepatosplenomegaly and thrombocytopenia was seen in $29.0 \%$. Azithromycin was used as first-line treatment in 56 (90.3\%) children while other children were treated with doxycycline and chloramphenicol. Seven (11.3\%) children required PICU admission. The mean duration of hospital stay for all children was $6.95( \pm 2.68)$ days while it was $9.00( \pm 3.51)$ days for children requiring PICU admission.

Conclusions: Scrub typhus should be considered in children with an acute febrile illness with hepatosplenomegaly and thrombocytopenia. Azithromycin is the drug of choice. Some children may need intensive care.

Keywords: Scrub typhus; Fever; Hepatosplenomegaly; Thrombocytopenia

\section{Introduction}

Scrub typhus is an acute febrile illness caused by obligate intracellular gram-negative bacterium Orientia tsutsugamushi transmitted to rodents (primary host) and humans (accidental host) by the bite of the larval form (chigger) of trombiculid mite (leptotrombidium). Scrub typhus is endemic to a part of the world known as the "tsutsugamushi triangle" which extends from northern Japan and far-eastern Russia in the north to northern Australia in the south and to Pakistan in the west [1]. Nepal is within the "tsutsugamushi triangle" [1]. There were a few attempts to investigate scrub typhus in Nepal, however, there was no clear evidence of apparent outbreak (and fatality) of scrub typhus in Nepal before 2014. Three months after the devastating earthquake in Nepal, B.P. Koirala Institute of Health Sciences (BPKIHS), Dharan had alerted Epidemiology and Disease Control Division (EDCD) that children with fever and severe respiratory features had not been responding to usual course of treatment but later the condition was confirmed to be scrub typhus and thus outbreak of scrub typhus was officially confirmed in Nepal in 2015 [1]. The WHO identifies scrub typhus as a re-emerging disease in South-East Asia with a case fatality rate up to $30 \%$ if left untreated [2].

Scrub typhus can have varied manifestations and can be mild to severe in children. The disease usually presents as an acute febrile illness with typical eschar, generalized lymphadenopathy, rash and non-specific symptoms such as fever, headache, myalgia and cough [3]. The disease usually responds dramatically to antibiotics but the delay in diagnosis and initiation of appropriate treatment can result in severe complications such as acute respiratory distress syndrome, 
septic shock, multi-organ failure and death [4]. The recommended treatment regimen for scrub typhus is doxycycline for a minimum of 5 days and until the patient has been afebrile for at least 3 days [5]. Azithromycin (for children $<8$ years) and doxycycline (for children $>8$ years) have been recommended by EDCD, Nepal for treatment of scrub typhus [6]. There are limited studies regarding the incidence and clinical profile of scrub typhus in children, despite the epidemiological mention of children constituting up to half of scrub typhus cases in some regions [7]. There are recent reports of scrub typhus from various parts of Nepal that might be due to altered environmental factors that occurred after the 2015 earthquake [8]. In 2016, a total of 831 cases with 14 deaths were reported in Nepal with more than one third (36.4\%) of cases being below 20 years [1].

However, we couldn't find any published literature on the clinical profile of scrub typhus in children in Nepal. Thus, we conducted a retrospective study at Kanti Children's Hospital $(\mathrm{KCH})$, Kathmandu, the only government tertiary level pediatric hospital in Nepal to study the clinico-laboratory profile and therapeutic outcome of serologically confirmed scrub typhus in children.

\section{Methods}

This was a retrospective study conducted at the Kanti Children's Hospital, Nepal. The medical records of 62 patients aged less than 14 years with serologically diagnosed scrub typhus and who were admitted to the hospital between August 2016 and January 2018 were reviewed. Data on age at admission, gender, initial presenting symptoms, examination findings on admission, a drug used for treatment and outcome in regards to whether the children were discharged after completion of treatment, referred to intensive care unit or expired and duration of hospital stay were collected. Leukocytosis and leucopenia were defined as total leukocyte count more than $11,000 / \mathrm{mm}^{3}$ and less than $4,000 / \mathrm{mm}^{3}$ respectively. Thrombocytopenia and severe thrombocytopenia was defined as platelet count less than $1,50,000 / \mathrm{mm}^{3}$ and $50,000 / \mathrm{mm}^{3}$ respectively. Hyponatremia was defined as serum sodium less than $135 \mathrm{mEq} / \mathrm{L}$. Ethical clearance was obtained from the institutional ethical committee before the initiation of the study. Data was entered and analyzed using SPSS 20. The descriptive statistics of the study variables were expressed in frequency and percentage, and they were summarized with the help of mean \pm standard deviation.

\section{Results}

This study included 62 children who were serologically diagnosed to have scrub typhus. Of the 62 children, 32 (51.6\%) were female and $30(48.4 \%)$ were male. The age of the children on presentation ranged from 10 months to 14 years with a mean age of $7.7( \pm 4.01)$ years. The most common age group affected was between 6 to 10 years of age (35.5\%). Among the study population, $45 \%$ of children were from 9 districts of Nepal which were severely affected by the 2015 earthquake; most were from Dhading (14.5\%), Gorkha (9.7\%) and Chitwan (8.1\%) districts; and most of them were admitted in August (22.6\%), September (29.0\%) and October (24.2\%) (Table 1).

Table 1: Temporal variation of scrub typhus in children $\mathrm{n}(\%)$.

\begin{tabular}{|c|c|c|c|c|}
\hline Month/Year & 2016 & 2017 & 2018 & Total \\
\hline July & - & $4(6.5 \%)$ & - & $4(6.5 \%)$ \\
\hline August & $6(9.7 \%)$ & $8(12.9 \%)$ & - & $14(22.6 \%)$ \\
\hline September & $8(12.9 \%)$ & $10(16.1 \%)$ & - & $18(29.0 \%)$ \\
\hline October & $6(9.7 \%)$ & $9(14.5 \%)$ & - & $15(24.2 \%)$ \\
\hline November & $2(3.2 \%)$ & $5(8.1 \%)$ & - & $7(11.3 \%)$ \\
\hline December & $1(1.6 \%)$ & $2(3.2 \%)$ & - & $3(4.8 \%)$ \\
\hline January & - & - & $1(1.6 \%)$ & $1(1.6 \%)$ \\
\hline Total & $23(37.1 \%)$ & $38(61.3 \%)$ & $1(1.6 \%)$ & $62(100.0 \%)$ \\
\hline
\end{tabular}

The clinical features at the time of presentation are shown in Table 2. All children presented with fever and majority (69.4\%) had a fever for 7 to 14 days. Gastrointestinal symptoms were present in $43.5 \%$ of children with abdominal pain and vomiting seen in $25.8 \%$ and $21 \%$ respectively. A cough, headache, generalized body swelling and abnormal body movement were present in $24.2 \%, 14.5 \%, 14.5 \%$, and $3.2 \%$ respectively. Hepatomegaly, splenomegaly, hepatosplenomegaly, lymphadenopathy, and rashes were found in $53.2 \%, 38.7 \%$, $32.2 \%, 17.7 \%$, and $9.7 \%$ respectively.
The laboratory profiles at the time of presentation are shown in Table 3. Anemia was seen in $61.3 \%$ of children. Leukocytosis was seen in $40.3 \%$ of children while leucopenia was seen in $8.1 \%$. Thrombocytopenia was seen in $72.6 \%$ of children with $14.5 \%$ of children having severe thrombocytopenia. The combination of hepatomegaly, splenomegaly, and thrombocytopenia was seen in $29 \%$ of cases. Serum sodium level on admission was investigated in 56 children among which hyponatremia was found in $28.6 \%$ of children. CSF analysis proved meningitis was seen in $3.2 \%$ of children. Other coinfections with Scrub Typhus seen were Leptospirosis (3.2\%), 
Malaria (3.2\%), Enteric fever (1.6\%), Dengue (1.6\%) and Brucella (1.6\%).

At Kanti Children's Hospital, one among azithromycin, doxycycline, and chloramphenicol was used as specific treatment. Azithromycin was used as first-line treatment in 56 (90.3\%) children (Table 4), with other been treated with doxycycline (6.5\%) and chloramphenicol (3.2\%). Azithromycin as first-line treatment was used for 5 to 7 days in 55 (88.7\%) children, while only 1 child was treated with a single dose of azithromycin. Second line treatment with doxycycline and chloramphenicol due to no response with azithromycin was used in $2(3.2 \%)$ and $1(1.6 \%)$ children respectively (Table 4$)$. Other antibiotics in combination with the specific treatment were used in 58 (93.5\%) children. Admission to the Pediatric Intensive Care Unit (PICU) was required in 7 (11.3\%) children. Six out of 7 children requiring PICU admission had a fever for 7 days or more. Among children requiring PICU admission, all 7 had thrombocytopenia and 3 had severe thrombocytopenia. One child died in PICU due to disseminated intravascular coagulation with severe thrombocytopenia with refractory shock. The mean duration of hospital stay for all children was 6.95 ( \pm 2.68 ) days; while it was $9.00( \pm 3.51)$ days for children requiring PICU admission and $6.69( \pm 2.48)$ days for children not requiring PICU admission.

Table 2: Demographic and clinical profile of scrub typhus in children.

\begin{tabular}{|c|c|c|c|c|c|c|c|c|}
\hline \multicolumn{3}{|c|}{ Demographic data $(n=62)$} & \multicolumn{3}{|c|}{ Symptoms $(n=62)$} & \multicolumn{3}{|c|}{ Signs $(n=62)$} \\
\hline & No. & $\%$ & & No. & $\%$ & & No. & $\%$ \\
\hline \multicolumn{3}{|c|}{ Age in years } & Fever & 62 & 100 & Rashes & 6 & 9.7 \\
\hline$<1$ year & 1 & 1.6 & $<7$ days & 10 & 16.1 & Lymphadenopathy & 11 & 17.7 \\
\hline 1-5 year & 20 & 32.3 & $7-14$ days & 43 & 69.4 & Hepatomegaly & 33 & 53.2 \\
\hline $6-10$ year & 22 & 35.5 & $>14$ days & 9 & 14.5 & Splenomegaly & 24 & 38.7 \\
\hline$>10$ year & 19 & 30.6 & GI symptoms & 27 & 43.5 & Hepatosplenomegaly & 20 & 32.2 \\
\hline \multicolumn{3}{|l|}{ Gender } & Pain abdomen & 16 & 25.8 & & & \\
\hline Female & 32 & 51.6 & Vomiting & 13 & 21 & & & \\
\hline Male & 30 & 48.4 & Cough & 15 & 24.2 & & & \\
\hline \multicolumn{3}{|l|}{ Districts } & Headache & 9 & 14.5 & & & \\
\hline Dhading & 9 & 14.5 & Swelling of body & 9 & 14.5 & & & \\
\hline Gorkha & 6 & 9.7 & & & & & & \\
\hline Chitwan & 5 & 8.1 & & & & & & \\
\hline Other & 42 & 67.7 & & & & & & \\
\hline
\end{tabular}

Table 3: Laboratory profile of scrub typhus in children.

\begin{tabular}{|c|c|c|}
\hline Laboratory findings $(n=62)$ & No. & $\%$ \\
\hline Anemia & 38 & 61.3 \\
\hline \multicolumn{3}{|l|}{ Total leucocyte count } \\
\hline$<4000$ & 5 & 8.1 \\
\hline $4000-11000$ & 32 & 51.6 \\
\hline$>11000$ & 25 & 40.3 \\
\hline \multicolumn{3}{|l|}{ Platelet } \\
\hline$<50000$ & 9 & 14.5 \\
\hline $50000-100000$ & 16 & 25.8 \\
\hline $100000-150000$ & 20 & 32.3 \\
\hline$>150000$ & 17 & 27.4 \\
\hline Hyponatremia $(n=56)$ & 16 & 28.6 \\
\hline
\end{tabular}


Table 4: First line treatment used in scrub typhus relation to age $(n=62)$.

\begin{tabular}{|l|l|l|l|}
\hline Age & Azithromycin & Doxycycline & Chloramphenicol \\
\hline$\leq 8$ years & $34(54.8 \%)$ & 0 & 0 \\
\hline$>8$ years & $22(35.5 \%)$ & $4(6.5 \%)$ & $2(3.2 \%)$ \\
\hline
\end{tabular}

\section{Discussion}

Scrub typhus is an acute febrile illness characterized by widespread infection of vascular endothelial cells resulting in significant vascular compromise and ensuing end-organ damage, most often manifested in brain and lungs [5]. In this retrospective study, we describe the clinic-laboratory profile of pediatric scrub typhus at the only tertiary pediatric hospital in Nepal.

The mean age at presentation was 7.7 years with minimum age 10 months; in line with other studies which reported the mean age between 6.5 to 8.8 years $[7,9,10]$; however, the minimum age reported by Palanivel et al. was 60 days [4]. Most of the children (69.3\%) were below 10 years of age similar to observations by Bhat et al. [7]. The male to female ratio was 1.07:1 similar to results obtained by Gurunathan et al [2]. Kanti Children's Hospital, the only governmental tertiary level pediatric referral hospital in Nepal, receives patients from different parts of Nepal but the catchments areas are all the 2015 earthquake-affected districts. Among the 62 children included, 9 (14.5\%) and 6 (9.7\%) were from Dhading and Gorkha respectively, rest were from other 25 (out of total 77) districts of Nepal. Gorkha and Dhading are among the 14 districts of Nepal severely affected by the 2015 earthquake [11]. Though most cases among the study population were admitted between August to October, similar to those reported by Karki et al. [1] and Sharma et al. [12], further studies are required to explain the temporal variation of scrub typhus cases.

The clinical manifestations of scrub typhus in children are mostly non-specific and likely to be misdiagnosed. Fever was reported in all children in present study similar to observations by Palanivel et al. [4], Bhat et al. [7], Narayanasamy et al. [10] and Rajendran [9]. Majority of children had a fever of 7 to 14 days duration similar to observations by Bhat et al. [7], Narayanasamy et al. [10] and Gurunathan et al. [2]. Gastrointestinal symptoms including pain abdomen, vomiting and diarrhea occur in up to $40 \%$ of children at presentation [5]. Similar finding were found in the present study (43.5\%). The predominant gastrointestinal symptoms differentiate scrub typhus from other febrile illness like malaria, dengue, leptospirosis as observed by Aung-Thu et al. [13] and Liu et al. [14] in their studies, accounting to $65 \%$ to $80 \%$ gastrointestinal symptoms. Typical eschar was not documented in the study population. The presence of eschar is a valuable clinical clue in the diagnosis and can be seen in $7 \%$ to $68 \%$ of cases [5], however, absence doesn't rule out the disease [4]. The lower prevalence of eschar is common in patients with scrub typhus in
Asia as they are dark skinned [2]. We observed hepatomegaly and splenomegaly in $53.2 \%$ and $38.7 \%$ respectively, whereas it was between $70 \%$ to $98 \%$ and $54 \%$ to $88 \%$ respectively as observed by Bhat et al. [7], Palanivel et al. [4] and Narayanasamy et al. [10]. The number of patients with hepatomegaly outnumbered that with splenomegaly in most studies $[4,7,10]$. Lymphadenopathy has been reported in $23 \%$ to $93 \%$ scrub typhus cases [5], but it was seen in $17.7 \%$ of cases in this study.

Thrombocytopenia was a major finding observed in $72.6 \%$ of children in this study similar to results reported by Palanivel et al. [4]. The combination of thrombocytopenia, hepatomegaly, and splenomegaly was seen in $29 \%$ patients in our study which seems to be clinically significant, hence scrub typhus should be considered a differential diagnosis in patients presenting with fever with hepatosplenomegaly and thrombocytopenia. Severe thrombocytopenia was seen in $14.5 \%$ of children, results contrary to results found by Bhat et al. [7] (27.2\%) and Narayanasamy et al. [10] (3\%). Leukocytosis and leukopenia were observed in $40.3 \%$ and $8.1 \%$ respectively. Hyponatremia was seen $28.6 \%$ of children in the present study whereas Palanivel et al. [4] and Narayanasamy et al. [10] reported it in $18 \%$ and $40 \%$ respectively.

Azithromycin was used in most cases (90.3\%) irrespective of age in the study population. This could be due to the availability of azithromycin in the tablet as well as in suspension formulation which together increases compliance. Doxycycline was a commonly used the drug for scrub typhus in studies by Palanivel et al. [4] and Bhat et al. [7]. But clinical trials have shown that azithromycin could be as effective as doxycycline for scrub typhus requiring modestly higher doxycycline minimal inhibitory concentration [5]. Azithromycin was used for 5 to 7 days in the majority of cases in the present study. Though single dose of azithromycin for scrub typhus has been recommended, in the present context without reliable, rapid diagnostics, it is thought to be hazardous to use a single dose of azithromycin for undifferentiated febrile illness as scrub typhus in Nepal can be co-infected with other infections like leptospirosis, requiring longer treatment [15]. Ojha and Rayamajhi have suggested rifampicin as a potential option in the treatment of scrub typhus [15]. However, in a country like Nepal, where tuberculosis is also widespread, it might be more prudent to reserve rifampicin for the treatment of tuberculosis [15]. Rifampicin was not used for scrub typhus at $\mathrm{KCH}$.

Admission to PICU was required in $11.3 \%$ of children in this study while $39 \%$ of cases required PICU admission in the study by Rajendran [9]. All 7 children requiring PICU admission had thrombocytopenia with 3 having severe thrombocytopenia. The 
mortality rate in our study was $1.6 \%$ while it was between 0 to $12 \%$ reported in studies reported by Rajendran [9], Palanivel et al. [4] and Bhat et al. [7]. The mean duration of hospital stay for all children was 6.95 ( \pm 2.68 ) days which was similar to results obtained by Rajendran [9]. Six out for 7 children requiring PICU admission had a fever for 7 days or more and the mean duration of hospital stay for children requiring PICU admission was 9.00 ( \pm 3.51) days compared to 6.69 ( \pm 2.48) days for children not requiring PICU admission. This suggests that those children requiring PICU admission presented late and thus delay in presentation increases the duration of hospital stay and need of intensive care. Thus, early presentation and treatment might decrease the subsequent morbidity and even death, however further studies on a larger scale would be required to verify this.

\section{Conclusion}

When a child presents with acute febrile illness, hepatomegaly, splenomegaly, and thrombocytopenia, diagnosis of scrub typhus should be considered in the present context of Nepal. Since serology for scrub typhus is not widely available in rural parts of Nepal, empirical therapy with azithromycin can be started on clinical grounds, as delay in presentation and treatment would result in significant morbidity and even death.

\section{Limitation}

The study was performed at a tertiary referral hospital; therefore does not reflect the actual burden of scrub typhus in the community.

\section{Acknowledgment}

We are grateful to medical record staffs of Kanti Children's Hospital who helped for data collection during the study.

\section{References}

1. Karki KB, Acharya BP, Dhimal M, Aryal KK, Sharma GN, et al. (2017) Descriptive epidemiology of Scrub typhus in Nepal. Nepal Health Research Council.
2. Gurunathan PS, Ravichandran T, Stalin S, Prabu V, Anandan H (2016) Clinical profile, morbidity pattern and outcome of children with scrub typhus. Int J Sci Stud 4: 247-250.

3. Wang CC, Liu SF, Liu JW, Chung YH, Su MC, et al. (2007) Acute respiratory distress syndrome in scrub typhus. Am J Trop Med Hyg 76: 1148-1152.

4. Palanivel S, Nedunchelian K, Poovazhagi V, Raghunadan R, Ramachandran P (2012) Clinical profile of scrub typhus in children. Indian J Pediatr 79: 1459-1462.

5. Kliegman RM, Stanton BMD, St. Geme J, Schor NF (2015) Nelson Textbook of Pediatrics (20th Edn). Elsevier 2.

6. Acharya B, Sharma GN, Shakya G (2015) EDCD interim Guideline on Prevention and Control of Scrub Typhus.

7. Bhat NK, Dhar M, Mittal G, Shirazi N, Rawat A, et al. (2014) Scrub typhus in children at a tertiary hospital in North India: Clinical profile and complications. Iran J Pediatr 24: 387.

8. Nayak N (2016) Scrub typhus in Nepal. Nepal J Epidemiol 6: 563.

9. Rajendran A (2011) Scrub typhus in paediatric age group: A report from a tertiary care hospital. J Pediatric Sci 3.

10. Narayanasamy DK, Arunagirinathan AK, Kumar RK, Raghavendran VD (2016) Clinico-laboratory profile of scrub typhus-an emerging rickettsiosis in India. Indian J Pediatr 83: 1392-1397.

11. http://www.who.int/hac/donorinfo/ nepal_country_update_funding_request_may2015.pdf

12. Sharma PK, Ramakrishnan R, Hutin YJF, Barui AK, Manickam P, et al. (2009) Scrub typhus in Darjeeling, India: opportunities for simple, practical prevention measures. Trans R Soc Trop Med Hyg 103: 1153-1158.

13. Aung-Thu SW, Phumiratanaprapin W, Phonrat B, Chinprasatsak S, Ratanajaratroj N (2004) Gastrointestinal manifestations of septic patients with scrub typhus in Maharat Nakhon Ratchasima Hospital. Southeast Asian J Trop Med Public Health 35: 845-51.

14. Liu YX, Jia N, Suo JJ, Xing YB, Liu G, et al. (2009) Characteristics of pediatric scrub typhus in a new endemic region of northern China. Pediatr Infect Dis J 28: 1111-1114.

15. Basnyat B (2016) Aftershocks of scrub typhus in Nepal-Author's reply. Lancet Glob Health 4: e688. 